\title{
DEFECT DENSITY AND ELECTRICAL PROPERTIES OF VACUUM EVAPORATED COPPER FILMS FROM ANNEALING STUDIES OF ELECTRICAL RESISTANCE
}

\author{
K. NARAYANDAS, M. RADHAKRISHNAN and C. BALASUBRAMANIAN \\ Department of Physics, Madras University Autonomous Post-Graduate Centre, \\ Coimbatore - 641041 , India
}

(Received February 20, 1981; in final form September 10, 1981)

\begin{abstract}
Copper films $(210-1650 \AA)$ were deposited onto glass microslides by vacuum evaporation. The films were subjected to heat treatment at a constant rate and the variation of electrical resistance with temperature was measured. The defect density and activation energy were calculated for different thicknesses from the knowledge of the change in electrical resistance with temperature and time. The defect density, $F_{0}(E)_{\max }$, varies from 17.2 to $6.05 \mu \Omega \cdot \mathrm{cm} \mathrm{eV}^{-1}$ in the thickness range $210-1650 \AA$ whereas no appreciable variation in the activation energy is observed.

Thickness dependence of resistivity and temperature coefficient of resistivity (TCR) were studied in the above thickness range and the bulk resistivity and TCR were found to be $1.75 \mu \Omega \cdot \mathrm{cm}$ and $5.5 \times 10^{-3} \mathrm{~K}^{-1}$ respectively. Assuming the scattering coefficient to be zero, the mean free path of conduction electrons was estimated. From the knowledge of the bulk resistivity and mean free path, the Fermi surface area and the effective free electron density per atom were evaluated as $21.86 \times 10^{16} \mathrm{~cm}^{-2}$ and 0.92 respectively.
\end{abstract}

\section{INTRODUCTION}

The electrical conductivity of thin metal films differ markedly from that of the bulk specimen. This difference increases with decrease in film thickness. ${ }^{1,2}$ Earlier workers ${ }^{3-8}$ have reported the dependence of the resistivity and temperature coefficient of resistivity (TCR) of noble metals on thickness. During the atomistic condensation process of the film formation, numbers of lattice defects are incorporated, which depend upon the evaporation conditions. Thus the incorporated impurities and structural defects formed during deposition, reduce the electron mean free path in thin films, and contribute considerably to the enhancement of the electrical resistivity. By the process of annealing and ageing the defects in the films can be removed. A number of theories ${ }^{9-11}$ have been proposed to evaluate the defect density and activation energy and several authors ${ }^{12-19}$ have applied Vand's theory ${ }^{10}$ to metals and alloys. In the present investigation, using Vand's theory, the thickness dependence of defect density and activation energy have been studied on copper films.

The films of different thicknesses required for the present study were prepared by thermal evaporation of pure copper (99.99\% Balzar, Switzerland) onto well cleaned glass substrates at room temperature. During the evaporation the pressure was maintained as $2 \times 10^{-5}$ Torr. The maximum increase in the temperature during the deposition was always approxi- mately $4^{\circ} \mathrm{C}$. For measurements, the temperature of the film was increased at a constant rate $\left(68^{\circ} \mathrm{K} \mathrm{h}^{-1}\right)$. The variation of resistance with temperature was studied using in-situ measurements, for various thickness of the film. The film thickness was measured by using multiple beam interferometer and verified by electrical methods. Copper-constantan thermocouples were used to measure the temperature of the film.

\section{RESULTS AND DISCUSSION}

\section{a) Thickness Measurement by Electrical Method:}

Thickness measurements were determined by optical and electrical methods. ${ }^{15}$ The optical method of thickness measurement was based on the principle of interference (Fizeau fringes) and the electrical method on the theory of electrical conductivity of thin films developed by Fuchs and Sondheimer. ${ }^{20,21}$

The ratio of conductivities can be written as:-

$$
\sigma_{0} / \sigma=\sigma_{0} R_{\square} K l
$$

where $\sigma$ and $\sigma_{0}$ are the conductivities of the film and bulk metal respectively, $l$ the mean free path of the conduction electrons in the bulk metal and $K=t / l$, ( $t=$ thickness of the film) $R_{\square}$, the sheet resistance of the film is given by the relation 


$$
R_{\square}=\frac{R b}{L}
$$

where $R$ is the total resistance, $b$ the breadth and $L$ the length of the film.

Furthermore a plot between $\sigma_{0} / \sigma$ and $K$ can be drawn for various arbitrary values of $K$, since according to Sondheimer ${ }^{21}$ :-

$$
\sigma_{0} / \sigma=1+\frac{3}{8 K}(1-p)(\text { if } K>1)
$$

where $p$ is the scattering coefficient of electrons at the inside surface of the film.

However this equation has been successfully applied by various workers ${ }^{7,22}$ for lower thickness ranges. Making use of the above concept and assuming $p$, the scattering coefficient, zero in Eq. 2, a curve was drawn between theoretical values of $\sigma_{0} / \sigma$ and $K$. Given $l=400 \AA$, and $\sigma_{0}$ derived from Figure 6 (which gives $\rho_{0}=1.75 \mu \Omega \mathrm{cm}$ ), it was possible to plot Eq. 1 in terms of experimental values of $R_{\square}$ for various assumed values of $K$ from 0.1 to 4 . From the point of intersection of the two curves, Eqs. 1 and 2, the values of $K$ can be determined from which ' $t$ ' is cal- culated. The theoretical and experimental curves are given in Figure 1. The graphical values of $K$ and thickness $t$ along with the thickness estimated from the multiple beam interferometer are presented in Table I for comparison.

The change of electrical resistance with time was measured for a freshly deposited film and the decrease in resistance was very rapid for a short period and then slowed up. This behaviour was more pronounced for thinner films than for thicker films (Figures 2a and 2b) which may be due to the incorporation of a large number of defects in the lower thickness films when compared to higher thickness films. A similar tendency was observed by Reynolds and Stillwell ${ }^{23}$ for copper and silver films.

TABLE I

\begin{tabular}{lcl}
\hline $\begin{array}{ll}\text { Electrical method (from graph) } \\
K\end{array}$ & $\begin{array}{l}\text { Optical method } \\
(\mathrm{MBI}) \\
t(\AA)\end{array}$ \\
\hline 0.525 & 210 & 210 \\
1.500 & 600 & 610 \\
3.25 & 1210 & 1205 \\
4.075 & 1640 & 1650 \\
\hline
\end{tabular}

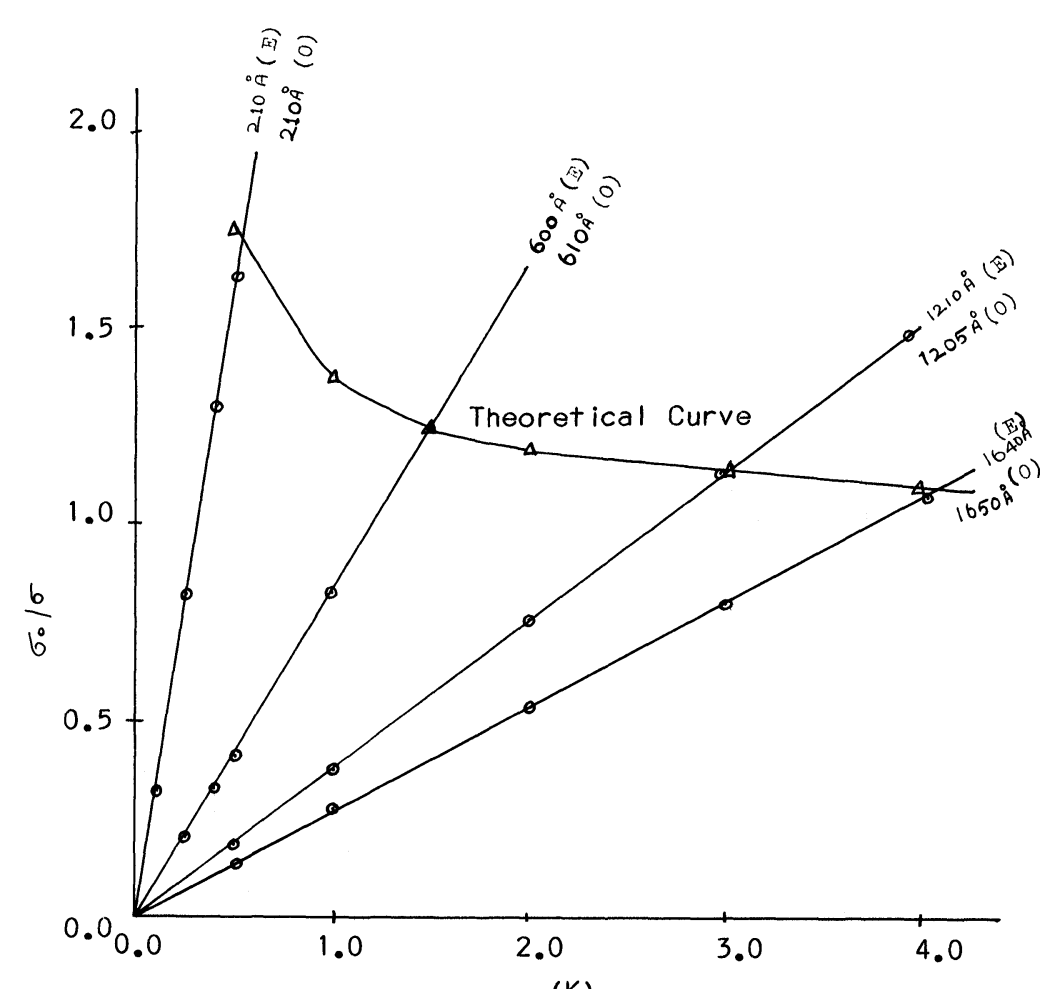

(K)

FIGURE 1 Curves of $\left(\sigma_{0} / \sigma\right)$ versus $K(=t / l)$ illustrating the method of calculating the film thickness from the conductivity values. ( $E$ : Electrical method; $O:$ Optical method) 


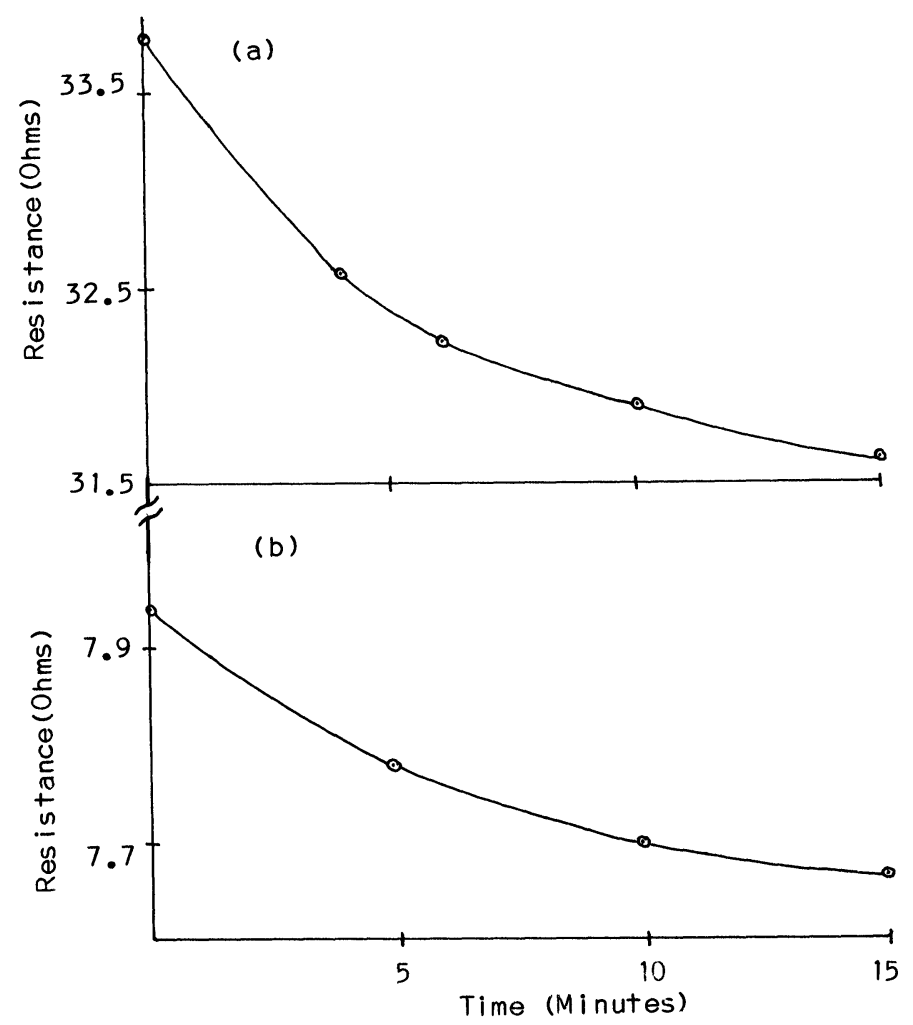

FIGURE 2 The variation of resistance with time for copper films of two different thicknesses (a) $210 \AA$ and (b) $430 \AA$.

\section{b) Dependence of Thickness on Defect Density:}

The resistance measurements were made as the temperature was raised and lowered at a constant rate $\left(68^{\circ} \mathrm{K} \mathrm{h}^{-1}\right)$ for various thicknesses $(210-1650 \AA)$ of copper film. Figures 3 and 4 show the variation of resistance with temperature during both heating and cooling cycles for different thicknesses. In the first cycle of annealing the nature of the variation of the resistance with the increase of temperature follows the general behaviour for most metals. The defects in the films disappeared slowly at first and then rapidly as the temperature increased. The nature of the variation of the curve depends upon the evaporation conditions. ${ }^{24}$ When the temperature was increased continuously, the resistance initially remains constant and then decreases. The rate of decrease was slow at first and then faster at higher temperatures. The observed decrease in resistance with temperature shows that the contribution to the resistance due to the removal of the defects is greater than that due to thermal vibration of the lattice. The temperature of the film was gradually increased to the annealing temperature, when the fall of resistance becomes a mini- mum, and then lowered. During the second cycle of heating and cooling the change of resistance with temperature was linear and retraceable i.e., the variation is purely due to the thermal vibration of the lattice.

Vand's theory ${ }^{10}$ was applied to our experimental data and the lattice distortion energy spectra were calculated for copper films of different thicknesses. Vand postulated that the distortions whose decay is observed during annealing are of the 'combined type', i.e., lattice vacancies and interstitials are in close proximity to one another. Independent vacancies and interstitials will need to diffuse large distances in the film to meet and annihilate one another or to migrate to the surface and these processes will require large activation energies. It is suggested that the contribution of independent vacancies and interstitials with regard to the decay observed during annealing is negligible. However the 'combined type' of distortions require only low activation energy, ${ }^{12,13,16,17,19}$ i.e., the defects will only need to diffuse over short distances. Therefore the observed irreversible resistance change in our investigation can reasonably by attribu- 


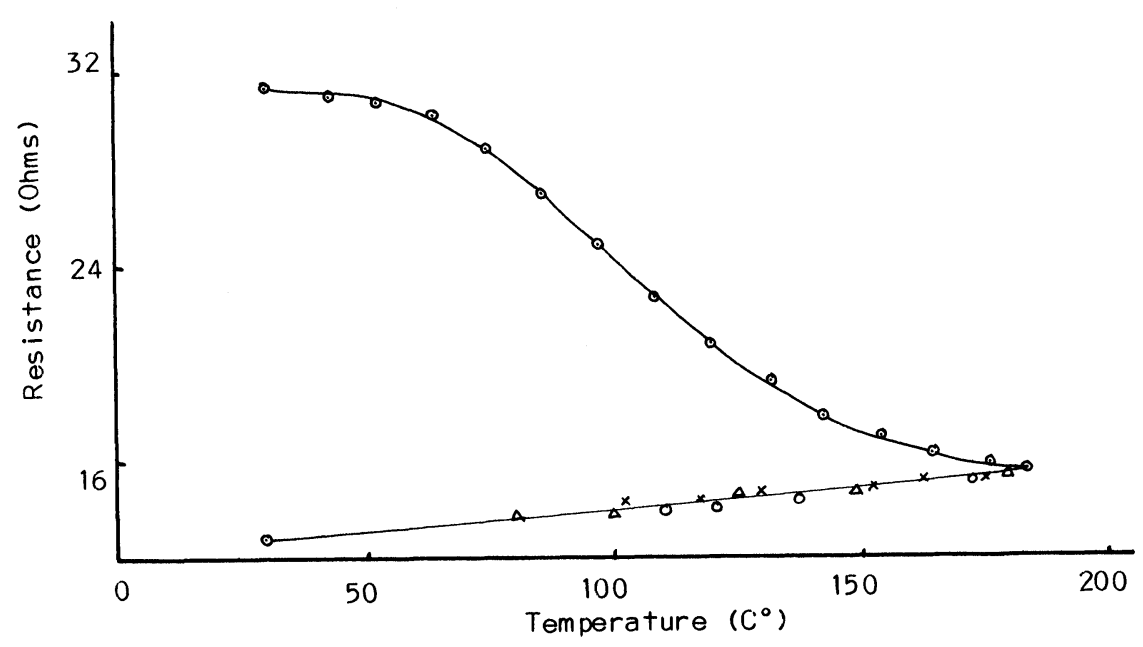

FIGURE 3 Resistance vs. temperature plot for copper film of thickness 210 A during heat treatment: $\odot$, x heating and cooling for cycle I; $\circ, \Delta$ for cyle II.

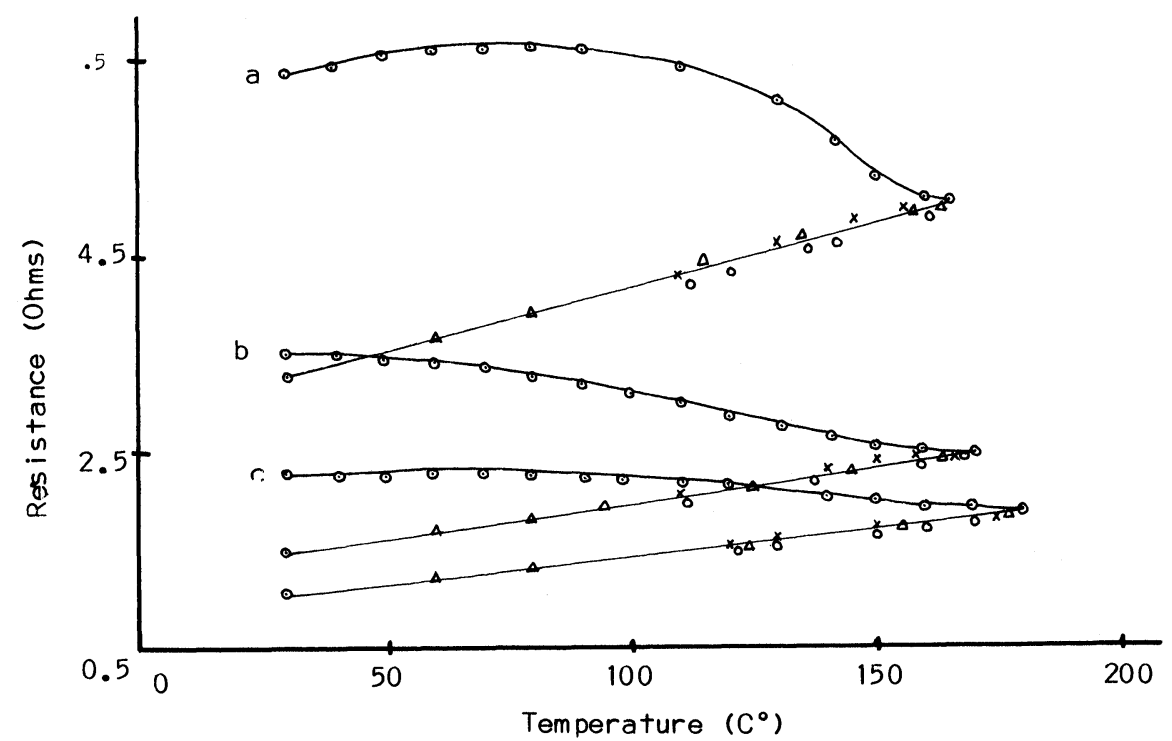

FIGURE 4 Resistance vs, temperature plots for copper films of different thicknesses (a) $610 \AA$ (b) $1205 \AA$ and (c) $1650 \AA$ during heat treatment: $\odot$, $\mathrm{x}$ heating and cooling for cycle $\mathrm{I}, \circ, \Delta$ for cycle II.

ted to the combined type of distortions. For the elimination of the combined type of defects, the required energy will be $\mathrm{E}$ which can vary from zero all the way to the activation energy for self-diffusion, depending on the structure of the defect.

If $\rho(E)$ is the contribution to the residual resistivity made by one distortion per unit volume and $N(E) \mathrm{d} E$ is the number of distortions per unit volume between decay energies $E$ and $E+\mathrm{d} E$, then the total contribu- tion made by all distortions is given by

$$
\rho_{i}=\int_{-\infty}^{+\infty} \rho(E) N(E) \mathrm{d} E
$$

where $\rho_{i}$ is the resistivity due to impurities which can change with time as a result of annealing. The distribution function $F_{0}(E)$ describing the initial energy 
spectrum of the distortions in the film is defined as $F_{0}(E)=\rho(E) N_{0}(E)$. From the analysis, Vand shows that

$$
F_{0}(E)=-\frac{1}{k U} \cdot \frac{\mathrm{d} \rho_{\mathrm{i}}}{\mathrm{d} T}
$$

where $k$ is the Boltzmann constant, $\mathrm{d} \rho_{\mathrm{i}} / \mathrm{d} T$ is the change in the impurity resistivity of the film with temperature during the first cycle of heating and $u$ is a function of decay energy $E(E=u k T)$ and the temperature $T . U$ can be defined as

$$
U=\frac{u(u+2)}{(u+1)}
$$

and $u+\log u=\log 4 n t \nu_{\max }$, where $\nu_{\max }=$ Debye out-off frequency for the lattice; $t=$ time taken to reach the particular temperature at which $\mathrm{d} \rho_{\mathrm{i}} / \mathrm{d} T$ is measured and $n=$ number of atoms that can initiate the decay of defect. The number $n$ can vary between wide limits from unity upwards. For the combined type Vand has estimated the value of ' $n$ ' to be of the order of 10 . By successively approximating the relation connecting $u$ and $4 n t \nu_{\max }$ and knowing the time $t, U$ can be calculated and hence from the experimental data, $F_{0}(E)$ and the activation energy $E$ can be obtained.

From Mattheissen's rule, $R=R_{i}+R_{T}$, where $R_{i}$ is the resistance due to defects and $R_{T}$ is that due to lattice vibration, $\mathrm{d} R_{i} / \mathrm{d} T$ can be calculated knowing $\mathrm{d} R / \mathrm{d} T$ from the first cycle of the heating curve and $\mathrm{d} R_{T} / \mathrm{d} T$ from the constant slope after annealing. The calculation of the change in the impurity resistivity with the change in temperature $\left(\mathrm{d} \rho_{\mathrm{i}} / \mathrm{d} T\right)$ during the first cycle of heating has been dealt with in detail elsewhere. ${ }^{16,17}$ Knowing the values of $\mathrm{d} \rho_{i} / \mathrm{d} T, U$ and $k$, both $F_{0}(E)$ and $E$ were calculated and a plot of $F_{0}(E)$ versus $E$ is drawn (Figure 5) for all the films.

The plot in Figure 5 shows that the distribution function maxima $F_{0}(E)_{\max }$ for the films vary from 17.2 to $6.05 \mu \Omega \cdot \mathrm{cm} \mathrm{eV}^{-1}$ and where the decay energy values of the defects vary between 0.94 to $1.59 \mathrm{eV}$ and the decay energies for which the $F_{0}(E)$ values is a maximum i.e. $E_{\max }$ varies over a range between 1.275 to $1.435 \mathrm{eV}$. As the thickness of the film increases coalescence of the island occurs and a continuous structure begins to form. At lower thickness the contribution of imperfections and impurities to the film resistivity becomes more important. Hence an increase of distribution function $F_{0}(E)_{\max }$ is observed for a decrease of film thickness. Since there is no appreciable variation of the activation energy with thickness, it is reasonable to say that the activation energy of a defect is the characteristics of the defects structure of the film lattice and it does not depend on the film thickness.

From the binding energy method as suggested by Vand, by knowing the melting point, boiling poin $\mathrm{t}^{25}$ and binding energy of copper, ${ }^{10}$ the approximate

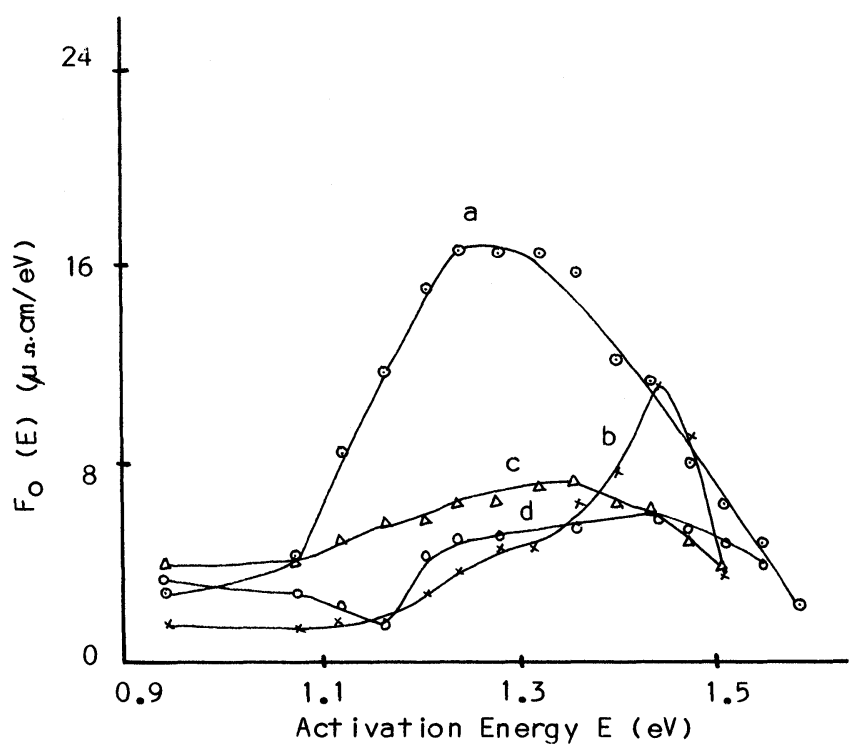

FIGURE 5 Lattice distortion energy spectra of copper films of different thicknesses: (a) $\odot-210 \AA$, (b) $x-610 \AA$, (c) $\triangle-1205 \AA$ and (d) $0-1650 \AA$. 
activation energy was calculated as $1.38 \mathrm{eV} .^{26}$ This is in good agreement with the experimentally observed average activation energy, $E_{\max }$ of $1.355 \mathrm{eV}$.

\section{c) Size Effect Study:}

The film boundaries play an important role in determining the film resistivity as the film thickness decreases, but its influence diminishes as the thickness increases. The plots of the resistivity and TCR of copper films $(210-1650 \AA)$ at room temperature $\left(30^{\circ} \mathrm{C}\right)$ against inverse thickness were drawn (Figures 6 and 7) and the experimental data were analysed using the expressions derived from size effect theory ${ }^{20,21}:-$

$$
\rho=\rho_{0}\left(1+\frac{3}{8} \frac{l(1-p)}{t}\right)
$$

and

$$
\alpha=\alpha_{0}\left(1-\frac{3}{8} \frac{l(1-p)}{t}\right)
$$

where $\rho$ and $\rho_{0}$, are the resistivities of the film and the bulk material, and $\alpha$ and $\alpha_{0}$, the TCR of the film and bulk material respectively. These equations are applicable for $t>1$. In a specific case of $t=210 \AA$, (i.e. $t>0.1$ ) the same expressions have been made use of as per the analysis done by earlier investigators. ${ }^{7,22}$ The above equations show a linear dependence of $\rho$ and $\alpha$ on inverse thickness of the film. The plots are found to be linear and by extrapolating the linear plots to infinite thickness, the resistivity and TCR

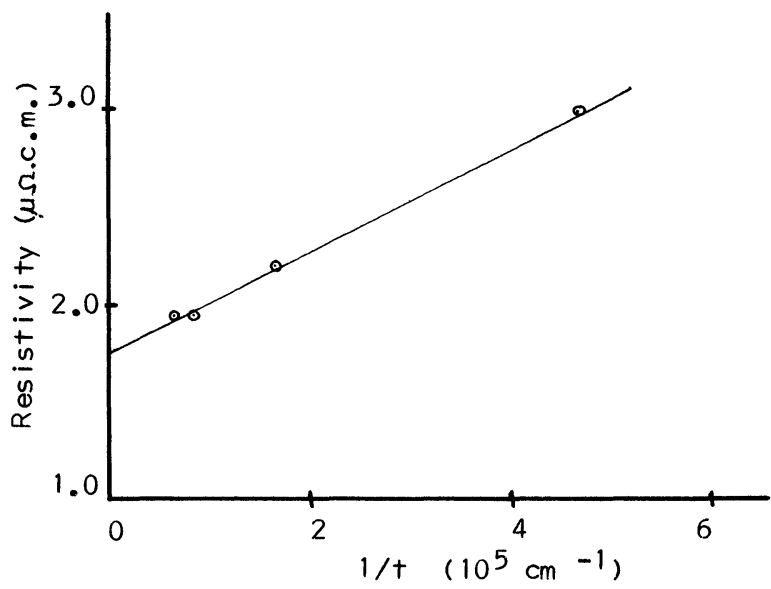

FIGURE 6 The variation of resistivity $(\rho)$ with inverse thickness $(1 / t)$ for copper films.

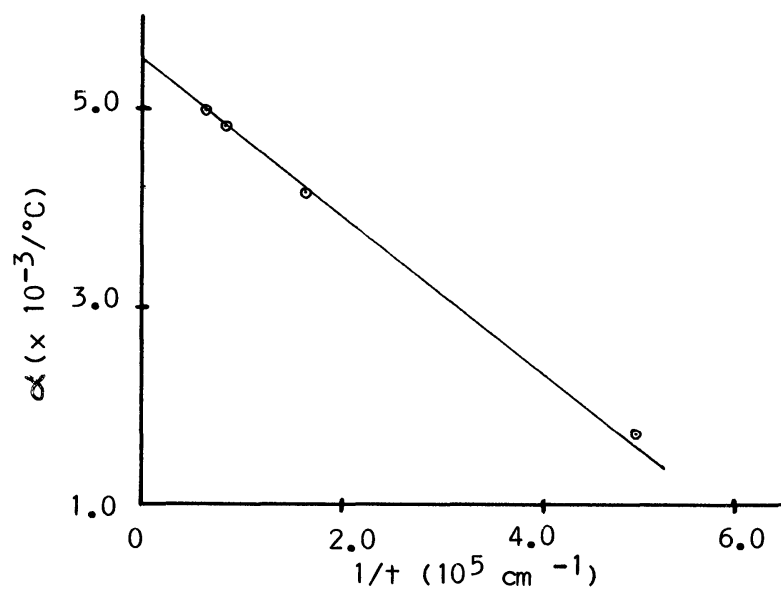

FIGURE 7 TCR $(\alpha)$ of Copper films as a function of inverse thickness $(1 / t)$.

corresponding to the bulk measurements have been evaluated to be $\rho_{0}=1.75 \mu \Omega \cdot \mathrm{cm}$ and $\alpha_{0}=5.5 \times$ $10^{-3} \mathrm{~K}^{-1}$ respectively. The slopes of the plots in Figures 6 and 7 is equal to $l(1-p)$. In order to determine the mean free path of the conducting electrons, the scattering coefficient $p$ needs to be evaluated from the measured resistivities and TCR of the films.

The values of $\rho / \rho_{0}$ and $\alpha / \alpha_{0}$ are piotted against $t / l$ (Figures 8 and 9 ). Assuming the film is to be polycrystalline in nature the theoretical curves of Fuchs and Sondheimer for $p=0$ were drawn (the continuous line in Figures 8 and 9) and the dotted line represents our experimental results. The curves are in fairly good agreement with each other, which means that the scattering coefficient $p$ is equal to zero or, in other

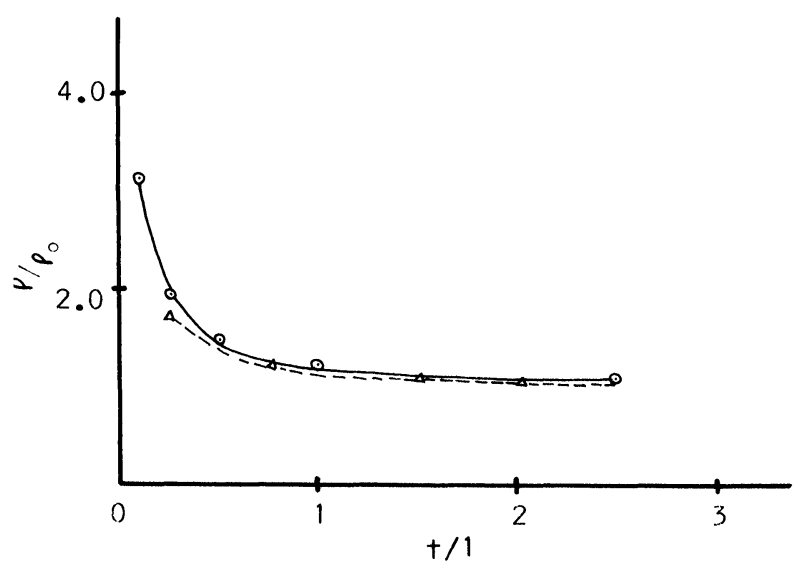

FIGURE 8 The variation of $\rho / \rho_{0}$ with $t / l$ for copper films. - - experimental; —_ theoretical. 


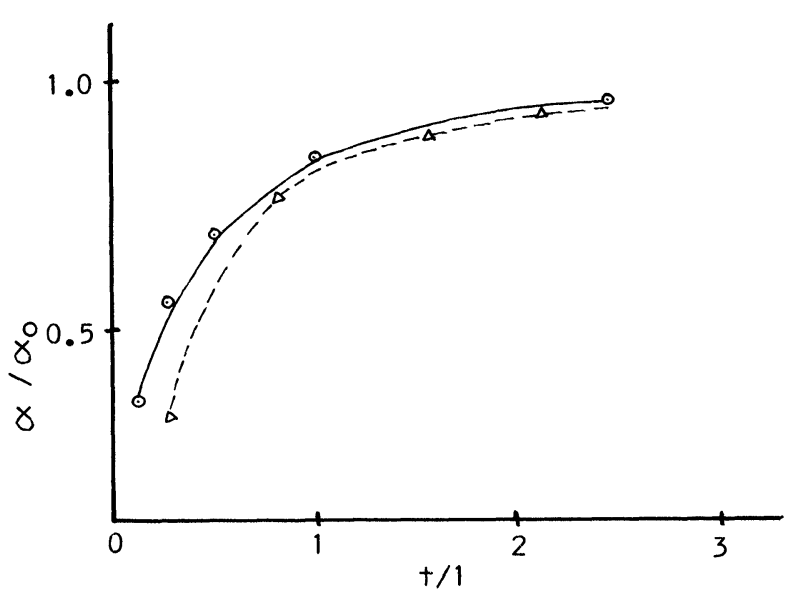

FIGURE 9 The variation of $\alpha / \alpha_{0}$ with $t / l$ for copper films. __- experimental; _— theoretical.

words, the scattering is perfectly diffuse for polycrystalline films. ${ }^{27,28}$ Substituting the values of $p=0$ from the slopes of the graphs of Figures 6 and 7, the means free path of the electrons were evaluated as $403 \AA$ and $397 \AA$ from the resistivity and TCR data respectively.

A plot of $\rho t$ versus $t$ was drawn (Figure 10) and from the point of intersection on the $\rho t$ axis, the mean free path of the electron was evaluated as $396 \AA$. The mean free paths evaluated from the various plots are in close agreement with the observa- tions made by Reynolds and Stillwell ${ }^{23}$ for polycrystalline films of copper.

Using Figure 10 the value of $\rho_{0} l$ has been determined as $0.73 \times 10^{-11} \Omega \mathrm{cm}^{2}$, whereas the value obtained by earlier workers ${ }^{23}$ for polycrystalline films of copper is $0.65 \times 10^{-11} \Omega \mathrm{cm}^{2}$. The slight difference in $\rho_{0} l$ may be due to experimental error.

From the value of $\rho_{0} l$, the Fermi surface area of copper was calculated using the relation ${ }^{29}$

$$
\frac{1}{\rho_{0} l}=\frac{e^{2} s}{12 \pi^{3} \hbar}
$$

where $s$ is the Fermi surface area, $e$ the charge on an electron and h, Planck's constant. The Fermi surface area was found to be $21.86 \times 10^{16} \mathrm{~cm}^{-2}$. The evaluated free electron density per atom is 0.92 which is in good agreement with that of the gold film (about 0.9 electron per atom) for $p=0 .{ }^{30}$

\section{CONCLUSIONS}

The change of electrical resistance with time for a freshly deposited film was more pronounced for thinner films than for thicker ones. It was found that the defect density of vacuum deposited copper films decreases with an increase of film thickness whereas there is no appreciable variation of the activation energy. The calculated bulk resistivity and TCR are

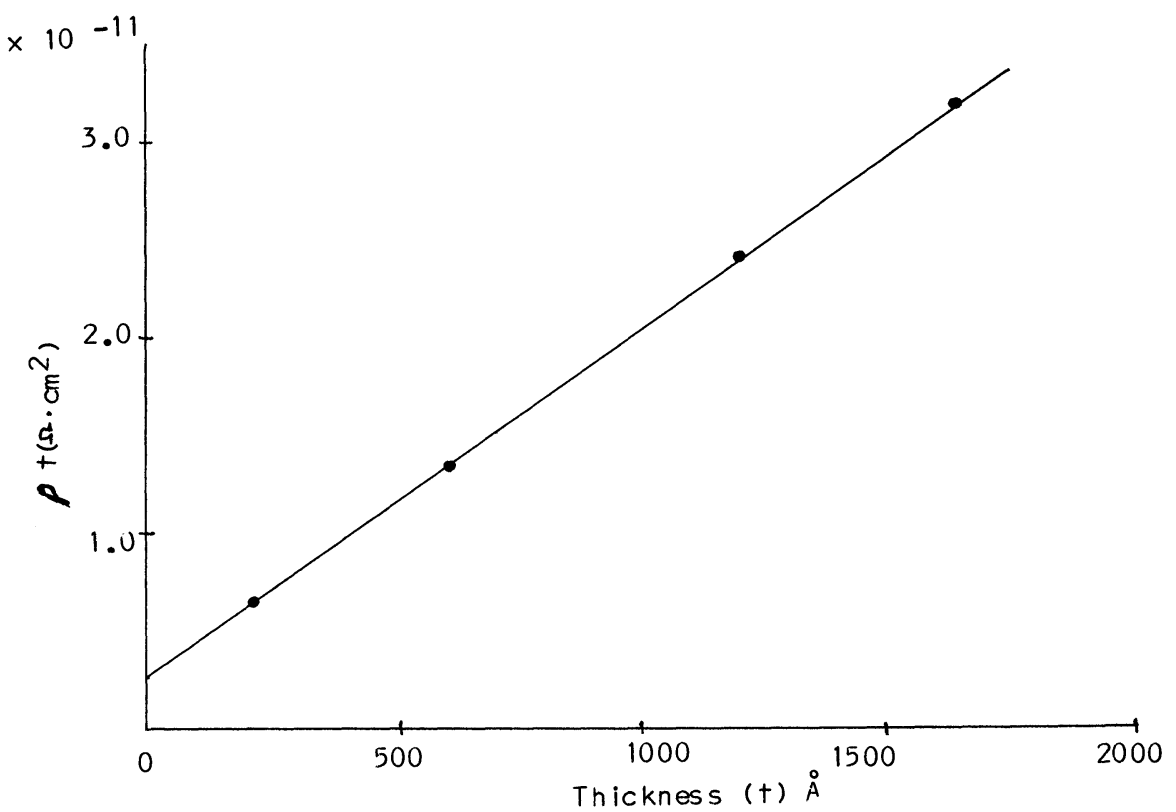

FIGURE 10 A plot of $\rho \mathrm{t}$ as a function of film thickness. 
in agreement with earlier observations. The theoretical curve agrees well with the experimental curve for $p=0$, and hence, considering the scattering to be perfectly diffuse, the mean free path of the conduction electrons evaluated from the resistivity and TCR data were $403 \AA$ and $397 \AA$ respectively. From the value of $\rho_{0} l$, the Fermi surface area and the electron density per atom were calculated as $21.86 \times 10^{16} \mathrm{~cm}^{-2}$ and 0.92 respectively.

\section{REFERENCES}

1. J.C. Anderson, The use of Thin Films in Physical Investigations (Academic Press, London, 1966).

2. T.J. Coutts, "Electrical conduction in Thin Continuous Films", Thin Solid Films, 7, 77 (1971).

3. K.L. Chopra, Thin Film Phenomena (McGraw-Hill, New York, 1969), Chap. VI, p. 381.

4. A.K. Pal, P. Sen and A.K. Barua, "Electrical properties of Vacuum Evaporated Copper Films", Thin Solid Films, 25, S25 (1975).

5. S.F. Lin and L.F. Leonard, "Thermoelectric power in Gold Films", J. Appl. Phys., 42, 3634 (1971).

6. W.F. Leonard and S.F. Lin, "The thermoelectric power to surface scattering in Thin Gold Films", Thin Solid Films, 11, 273 (1972).

7. V.V.R. Narasimha Rao, S. Mohan and P. Jayarama Reddy, "Electrical Resistivity, TCR and Thermoelectric power of annealed Thin Copper Films", J. Phys D: Appl. Phys. 9, 89 (1976).

8. S. Mohan and P. Jayarama Reddy, "Transport properties in annealed and agglomerated silver films", Nuclear Physics and Solid State Physics Symp., Bombay, India, 1974 in Solid State Physics, 17C, 128.

9. C.J. Meechan and J.A. Brinkman, "Electrical resistivity study of lattice defects introduced in coper by 1.25 Mev electron irradiation at $80 \mathrm{~K}$ ", Phys. Rev., 103, 1193 (1956).

10. V. Vand, "A theory of the irreversible electrical resistance changes of metallic films evaporated in vacuum", Proc. Phys. Soc., 55, 222 (1943).

11. W. Primak, "Kinetics of processes distributed in activation energy", Phys. Rev. 100, 1677 (1955).

12. P.G. Wilkinson, "Lattice distortion spectrum of evaporated Gold”, J. Appl. Phys., 22, 419 (1951).
13. P.G. Wilkinson and L.S. Birks, "Properties of Gold Deposited at liquid air temperature", J. Appl. Phys., 20, 1168 (1949).

14. H. Troyoda and M. Nagashima, "Electrical resistivity of arc-evaporated carbon films", J. Phys. Soc. Jpn., 14, 274 (1959).

15. V.V. Shah and Y.G. Naik, "Electrical properties of thin films of Copper, Silver and Gold", Indian J. Pure Appl. Phys., 3, 20 (1965).

16. K. Narayandas, M. Radhakrishnan and C. Balasubramanian, "Annealing Study of Electrical Resistivity and Defect Density in Silver Films", Phys. Status Solidi (a), 48, K71 (1978)

17. K. Narayandas, M. Radhakrishnan and C. Balasubramanian, "Thickness Dependence of Defect Density in Silver Films", Thin Solid Films, 67, 357 (1980).

18. L. Olumekar and J. Beynon, "Lattice distortion energy of heat-treated magnese films", Thin Solid Films, 53, L13 (1978).

19. V. Damodara Das, and M.S. Jagadeesh, "Annealing and thickness Effects on the Electrical Resistance of Vacuum Evaporated Tin antimonide alloy films", Thin Solid Films, 24, 203 (1974).

20. K. Fuchs, "Electron Theory of the Conductivity of Thin Metallic Films", Proc. Cambridge Philos., Soc. 34, 100 (1938).

21. E.H. Sondheimer, "The Mean Free Path of Electrons in Metals", Adv. Phys., 1, 1 (1952).

22. K.L. Chopra and M.R. Randlett, "Influence of a Super imposed film on the Electrical Conductivity of Thin Metal Films", J. Appl. Phys., 38, 3144 (1967).

23. F.W. Reynolds and G.R. Stillwell, "Mean Free Paths of Electrons in Evaporated Metal Films", Phys. Rev., 88, 418 (1952).

24. W. Romanvski and D. Potoczna - Petru, "The ageing of Thin Films of Nickel, Cobalt alloys in Ultra high vacuum", Thin Solid Films, 8, 35 (1971).

25. Robert C. Weast (Ed.), CRC Hand book of Physics and Chemistry (CRC Press, Ohio, USA, 1976-77) p. CPB-109.

26. K. Narayandas, M. Radhakrishnan and C. Balasubramanian, "Activation Energies of Copper and Silver Films", Indian Science Congress, India (1980).

27. A.K. Pal, U.De and P. Sen, "Resistivity of Polycrystalline Silver Films", Phys. Status Solidi (a) 27, K1 (1975).

28. S. Chandhuri and A.K. Pal, "The Resistivity and Temperature Coefficient of Resistivity of Polycrystalline lead Films", J. Phys. D: Appl. Phys., 8, 1311 (1975).

29. D.C. Larson, Physics of Thin Films, (Academic Press, New York, 1971) Vol. 6, p. 81-149.

30. K.G. Kedereit, "Untersuchungen Zur Elektrischen Leifa highkeit Von Dimen Gold-und Nickelaufdam pfschichten", Thin Solid Films, 1, 109 (1967). 

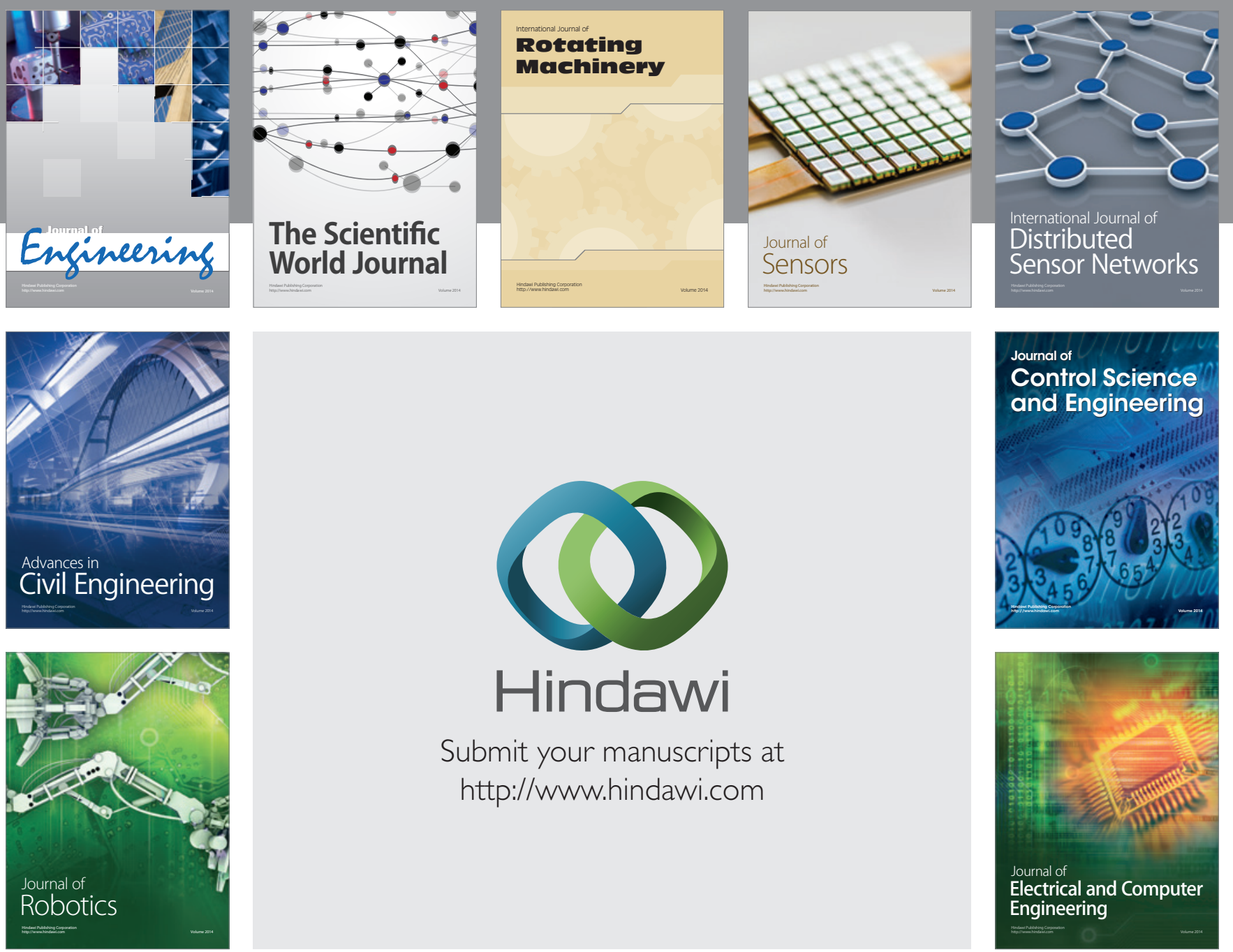

Submit your manuscripts at

http://www.hindawi.com
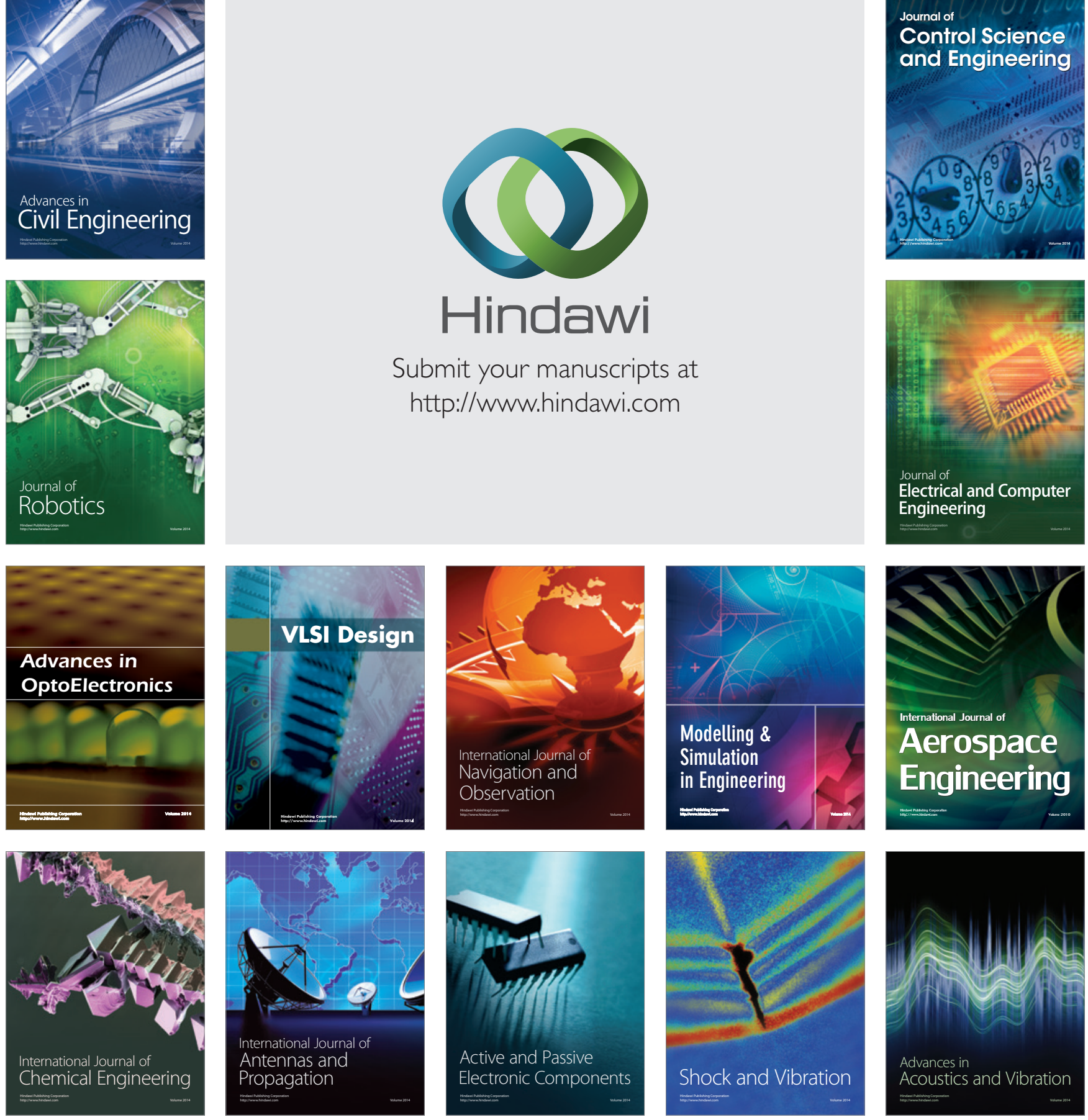